\title{
Induction dans l'expérience du monde et constitution du monde orienté de l'expérience en tant que monde avec terre et ciel
}

\section{Edmund Husserl}

Traducteur : Julien Farges et Laurent Perreau

\section{OpenEdition}

\section{Journals}

Édition électronique

URL : http://journals.openedition.org/alter/666

DOI : $10.4000 /$ alter.666

ISSN : 2558-7927

\section{Éditeur :}

Association ALTER, Archives Husserl (CNRS-UMR 8547)

\section{Édition imprimée}

Date de publication : 31 décembre 2018

Pagination : $231-240$

ISBN : 978-2-9550449-4-0

ISSN : 1249-8947

\section{Référence électronique}

Edmund Husserl, «Induction dans l'expérience du monde et constitution du monde orienté de l'expérience en tant que monde avec terre et ciel », Alter [En ligne], 26 | 2018, mis en ligne le 31 décembre 2019, consulté le 25 mars 2020. URL : http://journals.openedition.org/alter/666 ; DOI : https://doi.org/10.4000/alter.666 


\title{
INDUCTION DANS L'EXPÉRIENCE DU MONDE ET CONSTITUTION DU MONDE ORIENTÉ DE L'EXPÉRIENCE EN TANT QUE MONDE AVEC TERRE ET CIEL
}

\author{
Edmund Husserl
}

\section{Avant-propos des traducteurs}

Le texte qu'on va lire est extrait du tome XXXIX des Husserliana ${ }^{1}$. Celui-ci rassemble un grand nombre de manuscrits husserliens dédiés à l'exploration phénoménologique du monde de la vie ${ }^{2}$. Cet «appendice » a pour principal mérite d'illustrer une conception de la nature que Husserl développe dans différents textes datant de la dernière période de son œuvre. Il s'offre ici, sous la perspective particulière qui est celle de ce numéro de la revue Alter, comme un échantillon d'analyses plus développées et plus diversifiées, présentes dans différents volumes des Husserliana ${ }^{3}$. Ces différentes analyses promeuvent une conception nouvelle de la nature : celle-ci n'est plus l'ensemble des choses physiques situées dans l'espace et le temps, mais bien plutôt une structure générale de l'expérience qui s'étend infiniment depuis mon monde environnant, qui est commune à l'ensemble des hommes et qui, enfin, se caractérise par une certaine concrétude, pour peu qu'on

\footnotetext{
${ }^{1}$ E. Husserl, «Induktion in der Welterfahrung und die Konstitution der orientierten Erfahrungswelt als Welt mit Erde und Himmel », in Die Lebenswelt. Auslegungen der vorgegebenen Welt und ihrer Konstitution. Texte aus dem Nachlass (1916-1937), R. Sowa (éd.), Dordrecht, Springer, 2008, Husserliana XXXIX, Beilage XIII, p. 184-189.

${ }^{2}$ Nous renvoyons à notre recension: J. Farges, L. Perreau, «E. Husserl, Die Lebenswelt... », in Alter. Revue de phénoménologie, $\mathrm{n}^{\circ}$ 20, 2012, p. 213-222.

${ }^{3}$ Mentionnons le texte ${ }^{\circ} 4$ du Husserliana XXIX, daté de l'automne 1934 (p. 37-46), qui se présente comme une recherche relative aux "diverses formes d'historicité » et qui développe une « démythification (Entmythisierung) [du] premier monde objectif » (§4), ou encore le texte $\mathrm{n}^{\circ} 11$ (1930 ou 1931) du Husserliana XV, p. 157 sq.
} 
la dépouille $\mathrm{du}$ "vêtement d'idées » dont l'a recouverte la science moderne. Cette nature infinie, commune et concrète est abordée sous la perspective d'une ontologie du monde de la vie, mais celle-ci ne se conçoit elle-même que depuis la perspective fondatrice d'une phénoménologie transcendantale, soucieuse de révéler les structures de la subjectivité transcendantale.

Malgré sa brièveté, ce manuscrit de recherche présente l'intérêt de fournir, dans sa première moitié, des précisions sur le cadre théorique au sein duquel une telle description de la nature prend place, à savoir la mise au jour de l'inductivité comme structure fondamentale de l'expérience. Par ce terme auquel il recourt de plus en plus fréquemment à partir de la fin des années vingt, Husserl cherche à rendre compte de l'expérience comme d'un processus dynamique et unifié malgré ses discordances internes locales, qui ne saurait donc se comprendre comme la succession d'une pluralité d'expériences juxtaposées. Au contraire, son dynamisme repose sur un principe interne de liaison des expériences les unes avec les autres que Husserl nomme « induction » et qu'il ne faut pas confondre avec le procédé logique du même nom qui est à l'œuvre dans les sciences objectivo-théoriques de la nature : l'homonymie est certes destinée à montrer qu'il s'agit dans les deux cas d'une façon de se fonder sur le donné actuel pour se donner une prise anticipée sur du donné inactuel, mais c'est tout le sens de la description husserlienne des structures du monde de la vie, telle qu'elle se développe dans ces années, que de montrer que l'efficacité de l'induction objectivo-logique repose sur l'induction intuitive, immanente à l'expérience, qui l'appelle en quelque sorte comme son prolongement théorique.

Contrepartie directe du caractère horizontal de la donation des objets dans l'expérience, cette induction intuitive se trouve ici décrite comme un processus complexe mêlant deux aspects fondamentaux et inséparables, l'anticipation et la vérification. Dans la mesure où tout objet de l'expérience est donné sous la forme d'un noyau d'actualité entouré d'un halo de potentialités horizontales internes (les changements et les modalisations de l'objet lui-même) ou externes (les autres objets et, ultimement, le monde lui-même, co-donné en arrière-plan de l'objet thématique), l'expérience ne saurait se réduire à l'enregistrement passif d'un noyau de présent perceptif actuel mais consiste au contraire à viser anticipativement, à l'occasion du donné actuel, un déroulement ultérieur possible de l'expérience dont le caractère typique est fondé passivement sur les acquis sédimentés de l'expérience passée, des souvenirs et des habitudes associatives. Mais cela signifie que toute expérience est simultanément confirmation ou infirmation intuitive 
d'une anticipation passée et que cette typicité n'est donc pas constituée une fois pour toutes mais ne cesse de s'affiner et de se renforcer au cours de l'expérience, justifiant dès lors de nouvelles anticipations intuitives accompagnées d'une présomption toujours plus forte. C'est ce dernier aspect que Husserl nomme dans ces pages la structure de « vérification » immanente à l'expérience qui, elle non plus, ne s'identifie pas à la vérification expérimentale au sein des sciences de la nature mais la précède et la fonde au sein de notre rapport préscientifique et intuitif aux objets et au monde. Dans le présent texte, Husserl insiste en outre sur le fait que cette vérification qui s'effectue passivement au cours de l'expérience peut également faire l'objet d'une décision active, comme lorsque j'inspecte un objet ou lorsque je me déplace pour «vérifier» si les choses sont telles que me le faisait anticiper ce qui m'en a été actuellement donné auparavant. Mais cette vérification active n'est-elle pas tenue en échec par le fait que l'anticipation inductive porte plus loin que notre capacité à la vérifier, que ce soit volontairement ou non ? En effet, si l'horizontalité de l'expérience conduit, de proche en proche, à anticiper intuitivement la cohérence d'un monde ou la possibilité de faits naturels qui, à cause de leur éloignement spatial ou temporel, échappent à notre expérience, faut-il en conclure que cette dernière s'outrepasse elle-même de façon irrationnelle?

Les pages de ce manuscrit montrent que la phénoménologie a les moyens méthodiques de répondre à cette situation, par le biais d'une démarche constructive, c'est-à-dire qui étend la description, sur la base du donné intuitif, au-delà des limites de la donation (et donc de la vérification) intuitive. Construire, $c^{\prime}$ est ainsi chercher des vérifications et des confirmations intuitives indirectes, et parfois analogiques, en l'absence d'une expérience actuelle : ici, cette construction s'effectue en étendant au-delà de toute donation actuelle la structure proche-lointain telle qu'elle régit notre expérience perceptive courante. Dans ce cadre méthodologique, la nature se trouve donc thématisée comme le tout inductif et aperceptif de l'expérience perceptive, telle qu'elle implique le corps de chair comme organe de la perception.

Plus précisément, l'analyse phénoménologique de la «nature terrestre » fait apparaître celle-ci comme une totalité ouverte référée à un centre d'orientation. Elle est d'abord ce domaine limité qui fait l'objet d'un investissement culturel particulier, domaine qui est celui de ce que Husserl appelle, dans nombre d'autres textes de la même période, le territoire (Territorium). Mais Husserl ne s'attarde pas sur cet aspect qui le conduit souvent à revisiter la distinction classique nature/ culture. L'objet de l'analyse est ici la nature en tant qu'elle est aussi ce 
qui s'étend indéfiniment et continûment, au-delà du point de référence que constitue mon propre corps, en tant qu'il a une position particulière dans l'espace et le temps, dans la nature comme ensemble des corps. En ce sens bien précis, la nature est le monde commun appréhendé comme horizon externe de l'expérience. C'est sous cette perspective que Husserl esquisse une analyse de structures fondamentales qui régissent notre expérience de la nature, ainsi qu'un repérage d'éléments fondamentaux (soleil, lune, étoiles), qui sont comme des constantes de l'expérience de la nature.

On voit donc que si ce concept phénoménologique de la nature n'est évidemment pas celui de la science physico-mathématique exacte, il ne s'identifie pas non plus - il faut le souligner - à celui de la nature comme infrastructure fondatrice du monde réal, telle que la décrit l'ontologie matérielle déployée par exemple dans Ideen II. Il s'agit au contraire de cette nature que Husserl nomme parfois la nature vitalmondaine (lebensweltliche Natur), la nature telle qu'elle se laisse anticiper conformément aux structures qui régissent la constitution d'un monde préscientifique de l'expérience.

\section{$<$ Induction dans l'expérience du monde et constitution du monde orienté de l'expérience en tant que monde avec terre et ciel $>^{4}$}

La présupposition de la nature comme multiplicité définie ; tout énoncé [est] susceptible d'être fondé, immédiatement ou médiatement. L'opinion déterministe - le monde [comme] multiplicité définie ${ }^{5}$.

Etablir effectivement comme méthode l'anticipation (induction) et l'inductibilité qui a lieu dans la constitution aperceptive du monde de l'expérience - avec, comme noyau et à titre de vérification, la production de l'expérience possible anticipée - et, avant cela, rendre intuitif ce qui est anticipé, donc construire intuitivement une expérience possible comme [expérience] quasi-effective, mais alors nécessairement dans une généralité indéterminée en tant qu'espace de jeu des possibilités, conformément à l'appréhension typique qui se trouve dans l'aperception. En outre: capacité de [185] construire la forme ontologique du monde comme forme de son être-tel factuel possible, transfert du type univer-

${ }^{4}$ Sans doute 1934 [N.D.E.].

${ }^{5}$ De substances! 
sel dans une forme d'essence et application de la forme conceptuelle afin de systématiser ce dont il y a expérience dans son horizontalité formelle indéterminée et, par observation et expérimentation, de le déterminer méthodiquement, de façon toujours plus complète - conformément au jugement, dans une conceptualité exacte. Mais il manque ici le prolongement de ce qui a été dit précédemment. Face à un noyau comme celui de l'expérience directe, on peut " observer », on peut effectivement produire à titre de vérification ce qui est immédiatement induit au moyen d'une expérience directe qui progresse « de tous les côtés »; ou bien c'est une vérification négative qui survient (apparence), médiatisée par modalisation. C'est alors vers l'extérieur, conformément à l'horizon externe, que s'engage l'induction au second sens [du terme]. Une anticipation externe est donnée avec toute perception. De façon correspondante, toute "observation » faite sur place, tout développement d'une perception omnilatérale (donc: [toute] production des perceptions qui sont ici immédiatement induites intérieurement) donne lieu, correspondant à chaque nature, à une induction naturelle vers l'extérieur. "Sur place »- c'est-à-dire à l'intérieur de la continuité du percevoir, quelle que soit la façon dont il continue à s'écouler (mouvement oculaire, etc., mais aussi déplacement par locomotion).

En tant qu'elles sont rapportées à un monde environnant intersubjectif, les inductions externes se vérifient pour une part à travers mon propre déplacement actif et l'acte de percevoir correspondant, et comme toute vérification de ce genre (elle s'accomplit dans la continuité du percevoir), elle est exposée à un oui ou à un non; il y a aussi, chemin faisant, la possibilité intermédiaire que surviennent des modalisations «entre » l'être et le non-être. Pour une autre part, la confirmation s'accomplit par reprise des expériences étrangères dans une connexion immédiate ou bien par communication dans une expression documentée. Dans un cadre comme celui du présent concret, il est possible que l'étant qui persiste de façon inchangée soit aussi donné comme vérifié par le déplacement et la perception directe de ce qui a été précédemment exprimé et communiqué par des $<$ hommes $>$ et qui a été porté de façon persistante à la connaissance en tant qu'expression. Induction d'un élément passé : de façon immédiate par le souvenir propre. Mais ce dont il y a souvenir ne peut plus être observé de tous les côtés. On en reste alors à l'induction immédiate originaire des côtés qui sont demeurés inaperçus auparavant, dans leur déterminité relative et, pour le reste, leur généralité indéterminée. Il en va de même pour ce que les autres me communiquent (autres qui sont eux-mêmes induits à leur tour) ; ici, l'espace de jeu de l'indéterminité s'étend encore plus loin. De façon constante et nécessaire, il y a dans chaque 
validité ontologique l'évidence de l'activité de la vérifiabilité inductive selon le oui et le non, l'être et le non-être, de même aussi que celle des modalités de la validité.

Ayant un monde, je suis [1'] ego de mes capacités à porter l'induction mondaine à la vérification, ou encore à conduire activement l'induction - l'induction qui appartient à toute expérience du monde, qui en est constitutive - à des configurations [186] toujours nouvelles, et ce de telle sorte que s'accomplisse continuellement une vérification partielle grâce à la perception actuelle, et ensuite par la modification intentionnelle actuelle de la perception en tant qu'intuition faisant l'expérience. Cette dernière n'est que le noyau de la vérification. Car de façon corrélative, nous avons le jeu des inductions qui ne cessent d'être coéveillées; et à l'intérieur de ce cercle des [inductions] qui, dans leur particularité, demeurent non remplies (que ce soit de façon temporaire ou durable), c'est à présent une vérification ou une infirmation ellemême secondaire qui règne, selon que les inductions qui sont originairement éveillées ici ou là se remplissent, à leur manière en tant qu'anticipations expresses, d'un contenu déterminé (mais sans devenir intuitives), ou ne <se > remplissent pas. De même, l'expérience du monde est une expérience totale qui inclut ce qui, chaque fois, est horizon ainsi que les "préfigurations » déterminées en tant que composantes de sens saillantes et, eu égard à leur contenu, relativement " distinctes », jusqu'à l'horizontalité qui, au-delà, est encore vide. Tout cela dans un changement constant. Et cette intuition qui fait l'expérience $\mathrm{n}^{\prime}$ a sa validité ontologique intacte (elle n'est dans l'état de [l'intuition] vérifiante) que si le tout des préfigurations inductives qui, en tant que préfigurations extérieures, appartiennent à chaque expérience est un tout de la concordance. Mais pour ce qui est de l'expérience totale du monde, elle a une structure particulière. En tant que totalité, elle est dans une vérification constante pour autant qu'elle porte en elle l'évidence qu'une discordance singulière est décidable et qu'elle l'est de telle sorte que le tout soit totalement concordant.

Jusqu'où s'étend la vérifiabilité par l'expérience, jusqu'où s'étend l'induction et jusqu'où s'étend-elle en tant qu'elle est à vérifier ? Qu'en est-il, par exemple de ce qui est terrestre et qui doit encore être supposé extérieur à tout territoire humain ? Qu'en est-il des profondeurs de la Terre qui n'ont jamais été foulées? Comment le sens d'être "monde terrestre » se forme-t-il par constitution, lui qui dépasse la vérifiabilité actuelle et même vraisemblable ? Qu'en est-il de l'évidence de l'induction correspondante? Et si l'expérience nous fournit des motifs d'appréhender les faits célestes comme des faits de la nature, l'évidence n'est-elle pas ici du même type ? 
Le monde terrestre, dont il a été question jusqu'ici, est tout d'abord le monde environnant de mon peuple dans sa finitude territoriale, lequel $<a>$ toutefois autour de lui d'autres peuples dans les formes primitives de l'existence humaine, en expansion continue et ouverte sur le même sol naturel terrestre. La nature en tant que noyau signifie ici la nature terrestre, en tant qu'elle s'étend continûment, selon une progression que l'on peut réitérer, dans les directions d'orientation [définies] depuis la place actuelle que l'on occupe sur terre. Cela nous conduit tout d'abord à l'anticipation du sol terrestre, sur lequel s'offre la vie humaine, en tant que corporéité d'un genre spécifique, autonome, se continuant "infiniment», toujours et encore, précisément dans une expérience que l'on peut réitérer. Des corps délimités peuvent en être dissociés en tant qu'ils sont mobiles, conformément à l'expérience, et avec le sens [187] de la mobilité et de la variabilité, etc. Mais tous les corps délimités, les corps au sens habituel du terme, sont inclus en elle ; ils ont au-delà d'eux-mêmes leur espace libre, vide, qui leur appartient, dans lequel tous les corps peuvent se mouvoir, comme les oiseaux en vol. La nature terrestre a son espace naturel; elle a en outre son relief (les montagnes qui s'élèvent, etc.). Et l'air ? Il n'est tout d'abord pas distinct de l'espace. Il n'est pas un corps au sens habituel du terme, ni la terre au sens habituel du terme, sur laquelle les corps se tiennent et ainsi lui appartiennent, dont les corps pourraient se détacher, etc. L'air n'est pas la terre, il ne peut pas être morcelé (empiriquement parlant) en corps effectifs (fixes). Mais il est partout où il n'y a aucun corps. Pourtant il a en tant que vent, en tant que souffle, ses extensions avec leurs connexions et leurs positions, quand bien même ne sont-elles pas précisément déterminées. Il a une similitude avec l'eau, avec la vaste mer sans fin, dans laquelle on peut puiser et dont on peut faire pour soimême l'expérience, dans un récipient, en tant que corps - en tant que corps liquide. Mais en tout cas, si l'air est distinct de l'espace, il est aussi peu un corps, au sens habituel du terme, que l'est la terre une et sans fin qu'il entoure partout, dans un espace global, et qu'il remplit en tous ses creux ${ }^{6}$. Il appartient avec la terre (avec ce qu'il y a en elle de solide et de liquide), indissolublement, à un monde qui entoure tous les corps, tous les hommes et les animaux et qui les comprend tous en

\footnotetext{
${ }^{6}$ La tâche est d'abord de montrer comment advient l'aperception de la terre en tant que corps semblable à d'autres corps que nous ne pouvons pas embrasser du regard d'un seul coup (comme les montagnes). C'est ensuite seulement que se poserait la question de savoir à quel moment on est allé assez loin, dans le progrès de l'expérience, pour reconnaître le fait que toutes les voies terrestres reconduisent, de manière multilatérale, à leur point de départ. Mais l'aperception du vol des oiseaux et la représentation de la possibilité de pouvoir voler de plus en plus haut n'y suffit-elle pas?
} 
lui. Ce monde est le monde de l'espace; tout ce qui est corporellement fixe, <tout> ce qui est remarquable sous la modalité particulière de la forme aérienne et, naturellement, <tout $>$ ce qui est liquide, a sa position et sa forme actuelle et, dans cette forme persistante ou changeante, sa mobilité tout aussi bien que sa variabilité particulière.

Mais venons-en à présent au ciel et aux phénomènes célestes. Ma tentative en vue de leur reconnaître une modalité d'être spécifique (purement en tant qu'objets d'une expérience possible) n'est-elle pas une exagération? Les directions d'orientation, ou plus précisément les directions que je peux me représenter à partir de ma position actuelle, des corps en mouvement (les oiseaux en vol) fonctionnent comme si elles quittaient la surface terrestre vers l'avant, vers le ciel, et à chacune d'elles correspond une position dans le ciel. Entre la terre et le ciel, il y a l'espace aérien et le ciel lui-même a une forme empirique spatiale, celle d'une surface - mais, une fois encore, non pas celle d'une surface qui aurait un corps et non pas comme si elle avait la corporéité particulière de la terre.

Le ciel ne peut en aucune manière être vu corporellement et ainsi ce qui est «entre ciel et terre » n'est pas un espace intermédiaire comme un [188] espace entre deux choses ou un espace vide à l'intérieur d'une chose. D'un autre côté, tout comme une chose en recouvre subjectivement une autre et appartient, par ce recouvrement, un espace intermédiaire dont on peut faire expérience, chaque corps recouvre selon sa « situation par rapport au ciel » respective, un morceau de la surface céleste. En dessous d'elle, il y a l'espace en tant qu'espace aérien et <en tant qu'>espace rendant possible le mouvement, mais elle-même n'est ni spatiale, ni corporelle : ni spatiale, à savoir qu'elle n'est pas une surface dans l'espace - pour parler purement à partir de l'expérience. Quant à ce qui concerne à présent le soleil et la lune, ils ne sont pas véritablement appréhendés comme des corps. Ils appartiennent aussi au ciel mais ils ne sont pas vus comme des sphères ou des disques corporels. L'homme connaît les hautes montagnes, il connaît leur apparence diminuée par la perspective depuis des distances lointaines. Si ceci devait déterminer la perception du soleil et de la lune, alors les hommes se les représenteraient en tant que corps d'une dimension tout à fait exubérante. Mais le font-ils effectivement? Nous n'avons ici aucune aperception originelle pure et simple du lointain. Les choses en tant qu'elles sont situées loin de nous (par exemple, subjectivement, depuis un wagon) sont toujours $<$ données $>$ dans des aperceptions du lointain, ce sont continûment des phénomènes lointains, à partir de ce qui est familièrement proche (et relativement l'un par rapport à l'autre dans des modifications intentionnelles continues du à partir de..., à 
partir de..., à partir de...). Si elles disparaissent sous le cercle de l'horizon, si elles se ratatinent en un point comme une limite lointaine, alors cette limite n'est pas l'aperception du lointain, le phénomène du lointain depuis ce qui est familièrement proche, mais au contraire elle est justement comme le point-zéro de l'amoindrissement; <elle est> aussi peu une chose lointaine que le silence - qui est ce que le son anéantit - est un son. C'est seulement dans le passage à la limite que la limite reçoit son sens intentionnel en tant que limite du lointain (que l'on ne peut pas confondre avec la distance dans l'espace qui apparaît avec l'éloignement).

Comment en venons-nous donc à apercevoir la lune en tant que sphère ou disque lointain, en tant que corps lointain ? Tout ce qui est lointain sur terre l'est pour nous avant le ciel et le cercle de l'horizon sur terre (sur la mer par exemple) n'est pourtant pas au ciel. Entre les deux, il y a les nuages qui jouent, et qui recouvrent si souvent le ciel. Les nuages sont toujours devant le soleil, devant la lune. L'expérience conduit ainsi à considérer le ciel comme une surface de genre sphérique, sur laquelle la lune et les planètes se déplacent et sur laquelle les étoiles fixes ont une localisation immobile. Mais, au demeurant, le ciel reste le ciel et appréhender les étoiles comme des corps d'un lointain des plus lointains, c'est une prétention qui va bien au-delà du soleil et de la lune.

Dès lors, ces faits célestes représentent à nos yeux tout d'abord une sphère de faits spécifiques, purement à partir de leur expérience et de la confirmation empirique constante, dans leur "être ", une sphère de faits, c'est-à-dire des réalités existantes se confirmant dans leur sphère et se dénonçant éventuellement, dans le détail, comme des apparences. Mais seulement dans le détail. Car dans sa totalité l'être de la sphère céleste appartient continûment au monde des phénomènes, et selon une certitude se confirmant continûment. Le monde de l'expérience est toujours déjà [189] constitué en tant que monde terrestre, qui a, au sein de son phénomène universel, des corps en tant qu'étants sur le mode de la confirmation de ce qui est proche/lointain et en tant que monde céleste, qui a ses propres phénomènes universels (le ciel du jour et le ciel de la nuit, etc.) et ses propres étants individuels.

$<$ Ensuite $>$, le découpage de cette sphère ontologique incorporelle en apparitions lointaines de corps, par lesquelles on a donc l'expérience de corps en tant qu'ils sont pour nous, les sujets du monde environnant originel, et ensuite nécessairement en tant que corps dans un unique espace. Le monde, avec la duplicité des étants, devient un monde corporel avec les animaux qui lui appartiennent en tant que corps animés. Dans la progression de l'expérience : la lune dissimule les étoiles dans 
son mouvement, et on peut aussi à l'occasion observer que les planètes et les comètes dissimulent des étoiles fixes.

Mais en définitive, n'en va-t-il pas, avec l'aperception du lointain des corps célestes, en tant qu'elle n'est pas reconductible à l'expérience de ce qui est proche, tout autrement qu'avec les parties inaccessibles de la terre entre les territoires habités par des hommes ? Dans quelle mesure subsistent ici des différences transcendantalement constitutives?

Traduit de l'allemand par Julien Farges et Laurent Perreau 